\title{
Differentiability of Polynomials over Reals
}

\author{
Artur Korniłowicz \\ Institute of Informatics \\ University of Białystok \\ Poland
}

\begin{abstract}
Summary. In this article, we formalize in the Mizar system 3 the notion of the derivative of polynomials over the field of real numbers 4 . To define it, we use the derivative of functions between reals and reals 9 .
\end{abstract}

MSC: 26A24 03B35

Keywords: differentiation of real polynomials; derivative of real polynomials

MML identifier: POLYDIFF, version: 8.1.05 5.40.1286

\section{Preliminaries}

From now on $c$ denotes a complex, $r$ denotes a real number, $m, n$ denote natural numbers, and $f$ denotes a complex-valued function.

Now we state the propositions:

(1) $0+f=f$.

(2) $f-0=f$.

Let $f$ be a complex-valued function. Observe that $0+f$ reduces to $f$ and $f-0$ reduces to $f$.

Now we state the propositions:

(3) $c+f=(\operatorname{dom} f \longmapsto c)+f$.

(4) $f-c=f-(\operatorname{dom} f \longmapsto c)$.

(5) $c \cdot f=(\operatorname{dom} f \longmapsto c) \cdot f$.

(6) $f+(\operatorname{dom} f \longmapsto 0)=f$. The theorem is a consequence of (3).

(7) $f-(\operatorname{dom} f \longmapsto 0)=f$. The theorem is a consequence of (4). 
(8) $\square^{0}=\mathbb{R} \longmapsto 1$.

Proof: Reconsider $s=1$ as an element of $\mathbb{R} . \square^{0}=\mathbb{R} \longmapsto s$ by $[8$, (34)], [10, (7)].

\section{Differentiability of Real Functions}

One can check that every function from $\mathbb{R}$ into $\mathbb{R}$ which is differentiable is also continuous.

Let $f$ be a differentiable function from $\mathbb{R}$ into $\mathbb{R}$. The functor $f^{\prime}$ yielding a function from $\mathbb{R}$ into $\mathbb{R}$ is defined by the term

(Def. 1) $f_{\uparrow \mathbb{R}}^{\prime}$.

Now we state the propositions:

(9) Let us consider a function $f$ from $\mathbb{R}$ into $\mathbb{R}$. Then $f$ is differentiable if and only if for every $r, f$ is differentiable in $r$.

(10) Let us consider a differentiable function $f$ from $\mathbb{R}$ into $\mathbb{R}$. Then $f^{\prime}(r)=$ $f^{\prime}(r){ }^{1}$

Let $f$ be a function from $\mathbb{R}$ into $\mathbb{R}$. Observe that $f$ is differentiable if and only if the condition (Def. 2) is satisfied.

(Def. 2) for every $r, f$ is differentiable in $r$.

Let us note that every function from $\mathbb{R}$ into $\mathbb{R}$ which is constant is also differentiable.

Now we state the proposition:

(11) Let us consider a constant function $f$ from $\mathbb{R}$ into $\mathbb{R}$. Then $f^{\prime}=\mathbb{R} \longmapsto 0$. Proof: Reconsider $z=0$ as an element of $\mathbb{R} . f^{\prime}=\mathbb{R} \longmapsto z$ by $[9$, (22)], [10, (7)].

One can verify that $\operatorname{id}_{\mathbb{R}}$ is differentiable as a function from $\mathbb{R}$ into $\mathbb{R}$.

Now we state the proposition:

(12) $\quad \mathrm{id}_{\mathbb{R}}^{\prime}=\mathbb{R} \longmapsto 1$.

Proof: Set $f=\operatorname{id}_{\mathbb{R}}$. Reconsider $z=1$ as an element of $\mathbb{R} . f^{\prime}=\mathbb{R} \longmapsto z$ by [9, (17)], [10, (7)].

Let us consider $n$. One can verify that $\square^{n}$ is differentiable.

Now we state the proposition:

(13) $\left(\square^{n}\right)^{\prime}=n \cdot\left(\square^{n-1}\right)$.

From now on $f, g$ denote differentiable functions from $\mathbb{R}$ into $\mathbb{R}$.

\footnotetext{
${ }^{1}$ Left-side $f^{\prime}(r)$ is the value of the derivative defined in this article for differentiable functions $f: \mathbb{R} \mapsto \mathbb{R}$, and right-side $f^{\prime}(r)$ is the value of the derivative defined for partial functions in [9].
} 
Let us consider $f$ and $g$. Let us observe that $f+g$ is differentiable as a function from $\mathbb{R}$ into $\mathbb{R}$ and $f-g$ is differentiable as a function from $\mathbb{R}$ into $\mathbb{R}$ and $f \cdot g$ is differentiable as a function from $\mathbb{R}$ into $\mathbb{R}$.

Let us consider $r$. One can verify that $r+f$ is differentiable as a function from $\mathbb{R}$ into $\mathbb{R}$ and $r \cdot f$ is differentiable as a function from $\mathbb{R}$ into $\mathbb{R}$ and $f-r$ is differentiable as a function from $\mathbb{R}$ into $\mathbb{R}$ and $-f$ is differentiable as a function from $\mathbb{R}$ into $\mathbb{R}$ and $f^{2}$ is differentiable as a function from $\mathbb{R}$ into $\mathbb{R}$.

Now we state the propositions:

(14) $(f+g)^{\prime}=f^{\prime}+g^{\prime}$. The theorem is a consequence of (9) and (10).

(15) $(f-g)^{\prime}=f^{\prime}-g^{\prime}$. The theorem is a consequence of (9) and (10).

(16) $(f \cdot g)^{\prime}=g \cdot f^{\prime}+f \cdot g^{\prime}$. The theorem is a consequence of (9) and (10).

(17) $(r+f)^{\prime}=f^{\prime}$. The theorem is a consequence of (11), (3), (14), and (6).

(18) $(f-r)^{\prime}=f^{\prime}$. The theorem is a consequence of (11), (4), (15), and (7).

(19) $(r \cdot f)^{\prime}=r \cdot f^{\prime}$. The theorem is a consequence of (9) and (10).

(20) $(-f)^{\prime}=-f^{\prime}$.

\section{Polynomials}

In the sequel $L$ denotes a non empty zero structure and $x$ denotes an element of $L$.

Now we state the proposition:

(21) Let us consider a (the carrier of $L$ )-valued function $f$, and an object $a$. Then $\operatorname{Support}(f+\cdot(a, x)) \subseteq$ Support $f \cup\{a\}$.

Proof: $a=z$ or $z \in$ Support $f$ by [2, (32), (30)].

Let us consider $L$ and $x$. Let $f$ be a finite-Support sequence of $L$ and $a$ be an object. Observe that $f+\cdot(a, x)$ is finite-Support as a sequence of $L$.

Now we state the proposition:

(22) Let us consider a polynomial $p$ over $L$. If $p \neq \mathbf{0}$. L, then len $p-^{\prime} 1=$ len $p-1$.

Let $L$ be a non empty zero structure and $x$ be an element of $L$. Let us note that $\langle x\rangle$ is constant and $\left\langle x, 0_{L}\right\rangle$ is constant.

Now we state the proposition:

(23) Let us consider a non empty zero structure $L$, and a constant polynomial $p$ over $L$. Then

(i) $p=\mathbf{0} . L$, or

(ii) $p=\langle p(0)\rangle$. 
Let us consider $L, x$, and $n$. The functor $\operatorname{seq}(n, x)$ yielding a sequence of $L$ is defined by the term

(Def. 3) $\mathbf{0 .} L+\cdot(n, x)$.

Observe that $\operatorname{seq}(n, x)$ is finite-Support.

Now we state the propositions:

(24) $(\operatorname{seq}(n, x))(n)=x$.

(25) If $m \neq n$, then $(\operatorname{seq}(n, x))(m)=0_{L}$.

(26) the length of $\operatorname{seq}(n, x)$ is at most $n+1$.

(27) If $x \neq 0_{L}$, then len $\operatorname{seq}(n, x)=n+1$.

Proof: Set $p=\operatorname{seq}(n, x)$. For every $m$ such that the length of $p$ is at most $m$ holds $n+1 \leqslant m$ by (24), [1, (13)].

(28) $\operatorname{seq}\left(n, 0_{L}\right)=\mathbf{0} . L$. The theorem is a consequence of $(24)$.

(29) Let us consider a right zeroed, non empty additive loop structure $L$, and elements $x, y$ of $L$. Then $\operatorname{seq}(n, x)+\operatorname{seq}(n, y)=\operatorname{seq}(n, x+y)$. The theorem is a consequence of (24) and (25).

(30) Let us consider an add-associative, right zeroed, right complementable, non empty additive loop structure $L$, and an element $x$ of $L$. Then $-\operatorname{seq}(n, x)=\operatorname{seq}(n,-x)$. The theorem is a consequence of (24) and (25).

(31) Let us consider an add-associative, right zeroed, right complementable, non empty additive loop structure $L$, and elements $x, y$ of $L$. Then $\operatorname{seq}(n, x)-\operatorname{seq}(n, y)=\operatorname{seq}(n, x-y)$. The theorem is a consequence of $(30)$ and (29).

Let $L$ be a non empty zero structure and $p$ be a sequence of $L$. Let us consider $n$. The functor $p\lceil n$ yielding a sequence of $L$ is defined by the term

(Def. 4) $p+\cdot\left(n, 0_{L}\right)$.

Let $p$ be a polynomial over $L$. Let us note that $p\lceil n$ is finite-Support.

Let us consider a non empty zero structure $L$ and a sequence $p$ of $L$. Now we state the propositions:

(32) $\left(p\lceil n)(n)=0_{L}\right.$.

(33) If $m \neq n$, then $(p\lceil n)(m)=p(m)$.

Now we state the proposition:

(34) Let us consider a non empty zero structure $L$. Then $\mathbf{0 .} L \uparrow n=\mathbf{0} . L$. The theorem is a consequence of (32).

Let $L$ be a non empty zero structure. Let us consider $n$. One can verify that 0. $L \uparrow n$ reduces to $0 . L$.

Let us consider a non empty zero structure $L$ and a polynomial $p$ over $L$. Now we state the propositions: 
(35) If $n>$ len $p-^{\prime} 1$, then $p\lceil n=p$. The theorem is a consequence of (32).

(36) If $p \neq \mathbf{0} . L$, then len $\left(p\left\lceil\left(\operatorname{len} p-^{\prime} 1\right)\right)<\operatorname{len} p\right.$.

Proof: Set $m=\operatorname{len} p-^{\prime}$ 1. $m=\operatorname{len} p-1$. the length of $p\lceil m$ is at most len $p$ by [2, (32)], [7, (8)].

Now we state the proposition:

(37) Let us consider an add-associative, right zeroed, right complementable, non empty additive loop structure $L$, and a polynomial $p$ over $L$. Then $p\left\lceil\left(\operatorname{len} p-{ }^{\prime} 1\right)+\right.$ Leading-Monomial $p=p$. The theorem is a consequence of (32).

Let $L$ be a non empty zero structure and $p$ be a constant polynomial over $L$. Observe that Leading-Monomial $p$ is constant.

Now we state the proposition:

(38) Let us consider an add-associative, right zeroed, right complementable, distributive, unital, non empty double loop structure $L$, and elements $x$, $y$ of $L$. Then $\operatorname{eval}(\operatorname{seq}(n, x), y)=(\operatorname{seq}(n, x))(n) \cdot \operatorname{power}(y, n)$. The theorem is a consequence of (28), (27), and (25).

\section{Differentiability of Polynomials over Reals}

In the sequel $p, q$ denote polynomials over $\mathbb{R}_{\mathrm{F}}$.

Now we state the propositions:

(39) Let us consider an element $r$ of $\mathbb{R}_{\mathrm{F}}$. Then power $(r, n)=r^{n}$.

PRoOF: Define $\mathcal{P}$ [natural number] $\equiv \operatorname{power}\left(r, \$_{1}\right)=r^{\$_{1}}$. For every natural number $n, \mathcal{P}[n]$ from [1, Sch. 2].

(40) $\square^{n}=\operatorname{FPower}\left(1_{\mathbb{R}_{\mathrm{F}}}, n\right)$.

Proof: Reconsider $f=\operatorname{FPower}\left(1_{\mathbb{R}_{\mathrm{F}}}, n\right)$ as a function from $\mathbb{R}$ into $\mathbb{R}$. $\square^{n}=f$ by $[\underline{8}$, (36)], (39).

Let us consider an element $r$ of $\mathbb{R}_{\mathrm{F}}$. Now we state the propositions:

(41) $\operatorname{FPower}(r, n+1)=\operatorname{FPower}(r, n) \cdot \operatorname{id}_{\mathbb{R}}$.

(42) FPower $(r, n)$ is a differentiable function from $\mathbb{R}$ into $\mathbb{R}$.

Proof: Define $\mathcal{P}$ [natural number] $\equiv$ FPower $\left(r, \$_{1}\right)$ is a differentiable function from $\mathbb{R}$ into $\mathbb{R} . \mathcal{P}[0]$ by [6, (66)]. For every natural number $n$ such that $\mathcal{P}[n]$ holds $\mathcal{P}[n+1]$. For every natural number $n, \mathcal{P}[n]$ from [1, Sch. 2].

(43) $\operatorname{power}(r, n)=\left(\square^{n}\right)(r)$. The theorem is a consequence of (40).

Let us consider $p$. The functor $p^{\prime}$ yielding a sequence of $\mathbb{R}_{\mathrm{F}}$ is defined by

(Def. 5) for every natural number $n$, it $(n)=p(n+1) \cdot(n+1)$.

Note that $p^{\prime}$ is finite-Support.

Now we state the propositions: 
(44) If $p \neq \mathbf{0} \cdot \mathbb{R}_{\mathrm{F}}$, then len $p^{\prime}=\operatorname{len} p-1$.

Proof: Set $x=\operatorname{len} p-1$. Set $d=p^{\prime}$. the length of $d$ is at most $x$ by [7, (8)]. For every $n$ such that the length of $d$ is at most $n$ holds $x \leqslant n$ by [11, (7)], [7, (10)], [1, (21)].

(45) If $p \neq \mathbf{0} \cdot \mathbb{R}_{\mathrm{F}}$, then len $p=$ len $p^{\prime}+1$. The theorem is a consequence of (44).

(46) Let us consider a constant polynomial $p$ over $\mathbb{R}_{\mathrm{F}}$. Then $p^{\prime}=\mathbf{0} \cdot \mathbb{R}_{\mathrm{F}}$. The theorem is a consequence of (45).

(47) $(p+q)^{\prime}=p^{\prime}+q^{\prime}$.

(48) $(-p)^{\prime}=-p^{\prime}$.

(49) $(p-q)^{\prime}=p^{\prime}-q^{\prime}$. The theorem is a consequence of (47) and (48).

(50) Leading-Monomial $p^{\prime}=\mathbf{0} \cdot \mathbb{R}_{\mathrm{F}}+\cdot\left(\operatorname{len} p-^{\prime} 2, p\left(\operatorname{len} p-^{\prime} 1\right) \cdot\left(\operatorname{len} p-^{\prime} 1\right)\right)$.

Proof: Set $l=$ Leading-Monomial $p$. Set $m=\operatorname{len} p-^{\prime} 1$. Set $k=\operatorname{len} p-^{\prime} 2$. Reconsider $a=p(m) \cdot m$ as an element of $\mathbb{R}_{\mathrm{F}}$. Set $f=\mathbf{0} \cdot \mathbf{F}+\cdot(k, a) \cdot l^{\prime}=f$ by [1, (53)], [2, (31), (32)], [10, (7)].

(51) Let us consider elements $r, s$ of $\mathbb{R}_{\mathrm{F}}$. Then $\langle r, s\rangle^{\prime}=\langle s\rangle$.

Let us consider $p$. The functor $\operatorname{Eval}(p)$ yielding a function from $\mathbb{R}$ into $\mathbb{R}$ is defined by the term

(Def. 6) Polynomial-Function $\left(\mathbb{R}_{\mathrm{F}}, p\right)$.

Let us note that $\operatorname{Eval}(p)$ is differentiable.

Now we state the propositions:

(52) $\operatorname{Eval}\left(\mathbf{0} . \mathbb{R}_{\mathrm{F}}\right)=\mathbb{R} \longmapsto 0$.

Proof: $\operatorname{Eval}(\mathbf{0} . F)=\mathbb{R} \longmapsto 0(\in \mathbb{R})$ by $[5,(17)],[10,(7)]$.

(53) Let us consider an element $r$ of $\mathbb{R}_{\mathrm{F}}$. Then $\operatorname{Eval}(\langle r\rangle)=\mathbb{R} \longmapsto r$.

Proof: $\operatorname{Eval}(\langle r\rangle)=\mathbb{R} \longmapsto r(\in \mathbb{R})$ by [6, (37)], [10, (7)].

(54) If $p$ is constant, then $\operatorname{Eval}(p)^{\prime}=\mathbb{R} \longmapsto 0$. The theorem is a consequence of (23), (52), and (11).

(55) $\quad \operatorname{Eval}(p+q)=\operatorname{Eval}(p)+\operatorname{Eval}(q)$.

(56) $\operatorname{Eval}(-p)=-\operatorname{Eval}(p)$.

(57) $\operatorname{Eval}(p-q)=\operatorname{Eval}(p)-\operatorname{Eval}(q)$. The theorem is a consequence of (55) and (56).

(58) $\operatorname{Eval}($ Leading-Monomial $p)=\operatorname{FPower}\left(p\left(\operatorname{len} p-^{\prime} 1\right)\right.$, len $\left.p-^{\prime} 1\right)$.

Proof: Set $l=$ Leading-Monomial $p$. Set $m=\operatorname{len} p-^{\prime} 1$. Reconsider $f=$ FPower $(p(m), m)$ as a function from $\mathbb{R}$ into $\mathbb{R}$. $\operatorname{Eval}(l)=f$ by [5, (22)].

(59) $\quad$ Eval (Leading-Monomial $p)=p\left(\operatorname{len} p-^{\prime} 1\right) \cdot\left(\square^{\operatorname{len} p-^{\prime} 1}\right)$.

Proof: Set $l=$ Leading-Monomial $p$. Set $m=\operatorname{len} p-^{\prime} 1$. Set $f=p(m)$. $\left(\square^{m}\right) . \operatorname{Eval}(l)=f$ by (39), [8, (36)], [5, (22)]. 
(60) Let us consider an element $r$ of $\mathbb{R}_{\mathrm{F}}$. Then $\operatorname{Eval}(\operatorname{seq}(n, r))=r \cdot\left(\square^{n}\right)$. The theorem is a consequence of (24), (43), and (38).

(61) $\operatorname{Eval}(p)^{\prime}=\operatorname{Eval}\left(p^{\prime}\right)$.

Proof: Define $\mathcal{P}$ [natural number] $\equiv$ for every $p$ such that len $p \leqslant \$_{1}$ holds $\operatorname{Eval}(p)^{\prime}=\operatorname{Eval}\left(p^{\prime}\right) . \mathcal{P}[0]$ by $[5,(5)],(46),(52),(54)$. If $\mathcal{P}[n]$, then $\mathcal{P}[n+1]$ by $(36),[5,(3)],[1,(13)],(37) . \mathcal{P}[n]$ from [1, Sch. 2].

Let us consider $p$. Let us observe that $\operatorname{Eval}(p)^{\prime}$ is differentiable.

\section{REFERENCES}

[1] Grzegorz Bancerek. The fundamental properties of natural numbers Formalized Mathematics, 1(1):41-46, 1990.

[2] Grzegorz Bancerek and Andrzej Trybulec. Miscellaneous facts about functions Formalized Mathematics, 5(4):485-492, 1996.

[3] Adam Grabowski, Artur Korniłowicz, and Adam Naumowicz. Four decades of Mizar. Journal of Automated Reasoning, 55(3):191-198, 2015. doi 10.1007/s10817-015-9345-1

[4] Kazimierz Kuratowski. Rachunek różniczkowy i całkowy - funkcje jednej zmiennej. Biblioteka Matematyczna. PWN - Warszawa (in polish), 1964.

[5] Robert Milewski. The evaluation of polynomials Formalized Mathematics, 9(2):391-395, 2001.

[6] Robert Milewski. Fundamental theorem of algebra Formalized Mathematics, 9(3):461470, 2001.

[7] Michał Muzalewski and Lesław W. Szczerba. Construction of finite sequences over ring and left-, right-, and bi-modules over a ring, Formalızed Mathematıcs, 2(1):97-104, 1991.

[8] Konrad Raczkowski. Integer and rational exponents Formalized Mathematics, 2(1):125130, 1991.

[9] Konrad Raczkowski and Paweł Sadowski. Real function differentiability Formalized Mathematics, 1(4):797-801, 1990.

[10] Andrzej Trybulec. Binary operations applied to functions Formalized Mathematics, 1 (2):329-334, 1990.

[11] Michał J. Trybulec. Integers Formalized Mathematics, 1(3):501-505, 1990.

Received February 23, 2017 